The study of NCC effect on the state of oxidant/antioxidant balance in rats was indicative of the fact that according to the results obtained daily oral NCC administration during two weeks in the conditionally-effective dose $0,01 \mathrm{mg} / \mathrm{kg}(0,25 \%$ of $\mathrm{DL} 50)$, with the total course dose of $0,14 \mathrm{mg} / \mathrm{kg}$, was associated with inhibition of intensity of free radical lipid oxidation and protein oxidation modification (POM). It is evidenced by decreased content of TBARS (by $24,5 \%$ ) and POM in the blood plasma (by $38,6 \%$ ) in comparison with the control group of rats. Reduction of intensity of free radical lipid oxidation and POM occurs against the ground of activation of antioxidant protection system functioning in the animal body. Hence, during 14 days general antioxidant activity of the blood plasma in the blood of rats receiving NCC was higher than that of the control group. At the same time, the content of free $\mathrm{SH}$-groups in the blood plasma was $29,8 \%$ higher as compared with the control group.

The obtained results of our study concerning antioxidant effect of small NCC doses correlate with the literary data. Certain researchers (Iskra R.Y., 2013) found that addition of nanochromium citrate in the dose of $0,01 \mathrm{mg} / \mathrm{kg}$ to animal food increased activity of the major antioxidant enzymes - superoxide dismutase, catalase, glutathione peroxidase, glutathione reductase and reduced glutathione-SH in hemolysates of the blood erythrocytes. On the basis of the results of our own studies and literary data it can be suggested that antioxidant activity of MCC low doses is caused by activation of the antioxidant protection system - an increased activity of the key enzymes of the antioxidant protection in order to neutralize oxygen active forms and hydroperoxides.

Therefore, during long administration of NCC in the dose of $0,01 \mathrm{mg} / \mathrm{kg}$ the intensity of free radical lipid oxidation, POM content and TBARS decrease in the animal body against the ground of activation of the antioxidant protection system functioning.

\title{
Shchudrova T.S. \\ RENOPROTECTIVE EFFECT OF MELATONIN IN CONDITIONS OF ACUTE KIDNEY INJURY AND ALTERED PINEAL GLAND ACTIVITY
}

\author{
Department of Pharmacology \\ Higher State Educational Establishment of Ukraine \\ "Bukovinian State Medical University"
}

Multiple effects of melatonin make it a potential therapeutic agent. According to Ahmadian (2016), Reiter (2017) and Tavakoli (2014), melatonin, due to its free-radical scavenging activity and ability to potentiate the antioxidant system is a highly important antioxidant. Several researchers (Bonnefont-Rousselot, 2010; Espino, 2018; Esrefoglu, 2017; Ničković, 2018; Reiter, 2017) reported a therapeutic effect of melatonin in various pathologies related to oxidative stress. Besides, numerous studies (Majidinia, 2017; Pacini, 2016; Reiter, 2018; Tordjman, 2017) report beneficial immunostimulatory, anti-inflammatory, anti-apoptotic, cytoprotective, oncostatic, and anti-aging effects of melatonin.

The aim of this research was to study the renoprotective potential of melatonin in conditions of aminoglycoside-induced AKI against the background of pineal hypo- and hyperfunction.

Nonlinear mature white rats ( $\mathrm{n}=40$ ) were randomly divided into 5 groups. Animals from the I (Control), and II (AKI) group were kept under the natural light regimen. Pineal hypofunction was simulated in rats from the III group by maintenance under conditions of constant light at 500 lux (24.00 light : 0.00 darkness) for 7 days. Pineal hyperfunction was simulated in rats from the IV group by maintenance under conditions of constant darkness ( 0.00 light : 24.00 darkness). Toxic AKI (II-IV groups) was induced by daily administration of gentamicin at a dose of $80 \mathrm{mg} / \mathrm{kg}$ for 6 days. Animals from the III-IV groups were daily injected with melatonin at a dose of $5 \mathrm{mg} / \mathrm{kg} .24 \mathrm{~h}$ after the last injection biochemical and histological examination was performed. For the statistical analysis SPSS 17.0 software was used.

Nephrotoxicity of gentamicin caused significant $(\mathrm{p}<0.05)$ functional changes and structural alterations of rat kidneys. Treatment with melatonin in conditions of gentamicin-induced kidney injury significantly limited the degree of damage to renal tissue and prevented a critical reduction in kidney function, confirming a protective effect of melatonin. At the same time, significant $(\mathrm{p}<0.05)$ 
differences between the indices of the III and IV group allow us to state, that treatment with exogenous melatonin on the background of endogenous melatonin deficiency was less effective in comparison to the administration of melatonin in conditions of pineal hyperfunction.

Thus, melatonin ameliorates gentamicin-induced kidney injury by the limitation of histopathological changes in kidney tissue and preservation of kidney function. Pre-existing deficiency of endogenous melatonin decreases the resistance of kidneys to damaging action of the toxin and lessens the protective effect of the exogenous melatonin. Alternatively, in rats with increased pineal gland activity and melatonin production, co-treatment with exogenous melatonin more effectively protects the kidney from gentamicin-induced structural and functional changes and prevents the development of renal failure.

\section{Skrynchuk O. Ya. \\ STUDY OF SUGARS IN LEAVES OF CRAMBE KOKTEBELICA (JUNGE) N. BUSCH Department of Pharmacy \\ Higher State Educational Establishment of Ukraine \\ "Bukovinian State Medical University"}

Genus Crambe L. belongs to family Brassicaceae and has several dozen species, eight of which grow in Ukraine. Representatives of the studied genus are perennial and annual herbaceous plants originating from the subtropics (the Mediterranean, North and East Africa, and Central Asia). Crambe is widely used for environmental, food and technical purposes as a source of biofuel. In folk medicine, they are used in case of disorders of the digestive processes, as an anti-scurvy agent, as well as a substitute for mustard plasters.

Crambe koktebelica is of a particular interest. It is included in the list of species of flora that need special protection according to the Berne Convention (Convention for the protection of wild flora and fauna and natural habitats in Europe).

Crambe koktebelica (Junge) N. Busch is a local endemic species that grows singly or in small groups on limestone-gravelly slopes, on dry eroded clay slopes with washed away soils, on sea cliffs and shale deposits. In Ukraine, it is widespread in eastern Crimea. Local populations of Crambe koktebelica on the coast of Koktebel Bay and Karadag Mountain are known.

Analysis of scientific literature sources showed the lack of information on the chemical composition of species of the genus Crambe, including Crambe koktebelica (Junge) N. Busch, so the objective of our research was to study the qualitative composition and quantitative content of sugars in the leaves.

The material for experimental studies were the leaves of Crambe koktebelica, which were harvested during the mass flowering of plants in 2018 at the experimental sites of the Department of cultural flora of the M. M. Gryshko National Botanical Garden National Academy of Sciences of Ukraine in Kyiv.

The identification of this group of compounds was carried out by precipitation reaction using 96\% ethanol $\mathrm{P}$ and Fehling reagent after acid hydrolysis.

The qualitative composition and quantitative content of sugars in the studied object were studied by gas-liquid chromatography-mass spectrometry using chromatograph Agilent technologies $6890 \mathrm{n} / 5973$ inert chromatograph (USA). The identification of monosaccharides of the studied mixture was carried out by comparing the retention times of standard monosaccharides and using the NIST 02 mass spectrum library. Quantitative analysis was carried out by adding an internal standard solution to the test samples. Sorbitol solution was used as an internal standard.

The presence and the quantitative content of glucose $(19.02 \mathrm{mg} / \mathrm{g})$, galactose $(7.48 \mathrm{mg} / \mathrm{g})$, arabinose $(7.20 \mathrm{mg} / \mathrm{g})$, rhamnose $(3.81 \mathrm{mg} / \mathrm{g})$, mannose $(3.38 \mathrm{mg} / \mathrm{g})$, sucrose $(0.96 \mathrm{mg} / \mathrm{g})$ and fucose $(0.63 \mathrm{mg} / \mathrm{g})$ were established and determined in the composition of the polysaccharide complex of the object under study. The data obtained indicate that the leaves of Crambe koktebelica are promising raw materials for the development of new drugs. 